\title{
Overview of RoboCup-99
}

Manuela Veloso ${ }^{1}$, Hiroaki Kitano ${ }^{2}$, Enrico Pagello ${ }^{3}$, Gerhard Kraetzschmar ${ }^{4}$, Peter Stone ${ }^{5}$, Tucker Balch ${ }^{1}$, Minoru Asada ${ }^{6}$, Silvia Coradeschi ${ }^{7}$, Lars Karlsson ${ }^{7}$, and Masahiro Fujita ${ }^{8}$

${ }^{1}$ School of Computer Science, Carnegie Mellon University, Pittsburgh, USA

${ }^{2}$ Sony Computer Science Laboratories, Inc., Tokyo, Japan

${ }^{3}$ Dept. of Electronics and Informatics, The University of Padova, Italy

${ }^{4}$ Neural Information Processing, University of Ulm, Ulm, Germany

${ }^{5}$ AT\&T Labs - Research, 180 Park Ave., Florham Park, USA

${ }^{6}$ Adaptive Machine Systems, Osaka University, Osaka, Japan

7 Örebro University, Örebro, Sweden

${ }^{8}$ Sony Corp., Tokyo, Japan

\begin{abstract}
RoboCup-99, the third Robot World Cup Soccer Games and Conferences, was held in conjunction with IJCAI-99 in Stockholm. RoboCup has now clearly demonstrated that it provides a remarkable framework for advanced research in Robotics and Artificial Intelligence. The yearly RoboCup event has included a technical workshop and competitions in different leagues. This chapter presents a comprehensive overview of RoboCup-99 and the scientific and engineering challenges presented to the participating researchers. There were four RoboCup-99 competitions: the simulation league, the small-size robot league, the middle-size robot league, and, for the first time officially, the Sony legged robot league. The champion teams were CMUnited-99 (Carnegie Mellon University, USA) for the simulation league, Sharif CE (Sharif University of Technology, Iran) for the middle-size league, Big Red (Cornell University, USA) for the small-size league, and "Les 3 Mousquetaires" (Laboratoire de Robotique de Paris, France) for the Sony legged robot league. The Scientific Challenge Award was given to three papers on innovative research for the automated statistical analysis of the games, from the University of Southern California (ISI/USC), USA, the Electrotechnical Laboratory (ETL), Japan, and Chubu University, Japan. There will be the first RoboCup European Championship in Amsterdam in May 2000, and the International RoboCup-2000 will take place in Melbourne, Australia, in August 2000.
\end{abstract}

\section{Introduction}

The RoboCup Initiative, the Robot World Cup Soccer Games and Conferences, provides a large spectrum of research and development issues in Artificial Intelligence (AI) and Robotics. In particular, it remarkably provides a common task, namely robotic soccer, for the investigation and evaluation of different approaches, theories, algorithms, and architectures for multiagent software and robotic systems. 
RoboCup-99, held in Stockholm, followed the successful RoboCup-97 in Nagoya [6] and RoboCup-98 in Paris [3]. The RoboCup-99 event included a technical workshop, robotic soccer competitions in four different leagues, and a variety of demonstrations.

This chapter introduces the different leagues in detail, summarizes the challenging research problems underlying each league, and overviews RoboCup-99. It further includes several appendices with the results of all the games in the four competition leagues. It shows the results of the preliminary round-robin phases and the results from the elimination rounds. The book contains the technical contributions on how each of the multiple teams concretely addressed these research challenges at the RoboCup-99 competitions. The chapter includes a brief discussions of the lessons learned and future directions. A new RoboCup search and rescue task is under development and will be part of the next RoboCup events.

The RoboCup events are held every year. RoboCup has been held in conjunction with international technical conferences. It has been attended by the research community and by the general public. RoboCup-97 and RoboCup-99 were held at the biannual International Joint Conference on Artificial Intelligence (IJCAI). RoboCup-98 was held with the International Conference on Multiagent Systems (ICMAS) in Paris. RoboCup-98, in particular, attracted a large audience, as it took place mostly at the same time as the human World Cup.

RoboCup-99, the Third Robot World Cup Soccer Games and Conferences, was held on July 27th through August 4th, 1999 in Stockholm. It was organized by Linköping University with the cooperation of Stockholm University, and it was sponsored by Sony Corporation, Sun Microsystems, Futurniture, First Hotel, The Foundation for Knowledge and Competence Development, The Swedish Council for Planning and Coordination of Research, The Swedish Foundation for Strategic Research, NUTEK, and WITAS.

The purpose of RoboCup is to provide a common task for evaluation of different algorithms and their performance, theories, and robot architectures [8]. In addition, as soccer, as a game, is quite accessible to both experts and non-experts, RoboCup has also shown to provide an interesting popular demonstration of research in AI and Robotics.

RoboCup-99 had four different leagues, each one with its specific architectural constraints and challenges, but sharing the goal of developing teams of autonomous agents with action, perception, and cognition. RoboCup-99 also included the RoboCup Jr. event targeted at allowing children to experiment with automated robotic systems.

The Scientific Challenge Award is given each year to people or groups that have made significant scientific contributions to RoboCup. At RoboCup-99, the Scientific Challenge Award was given to three papers on innovative research for the automated statistical analysis of the games, from the University of Southern California (ISI/USC), USA, the Electrotechnical Laboratory (ETL), Japan, and Chubu University, Japan. 


\section{Simulation League}

The simulation league continues to be the most popular part of the RoboCup leagues, with 37 teams participating in RoboCup-99, which is a slight increase over the number of participants at RoboCup-98. In this section, we briefly describe the RoboCup simulator; we present the major research challenges and some of the ways in which they have been addressed in the passed; and we summarize the 1999 competition.

\subsection{The RoboCup Simulator}

The RoboCup-99 simulation competition was held using the RoboCup soccer server [11], which has been used as the basis for previous successful international competitions and research challenges [8]. The soccer server is a complex and realistic domain, embracing as many real-world complexities as possible. It models a hypothetical robotic system, merging characteristics from different existing and planned systems as well as from human soccer players. The server's sensor and actuator noise models are motivated by typical robotic systems, while many other characteristics, such as limited stamina and vision, are motivated by human parameters.

The simulator includes a visualization tool, pictured in Figure 1. Each player is represented as a two-halved circle. The light side is the side towards which the player is facing. In Figure 1, all of the 22 players are facing the ball, which is in the middle of the field. The black bars on the left and right sides of the field are the goals.

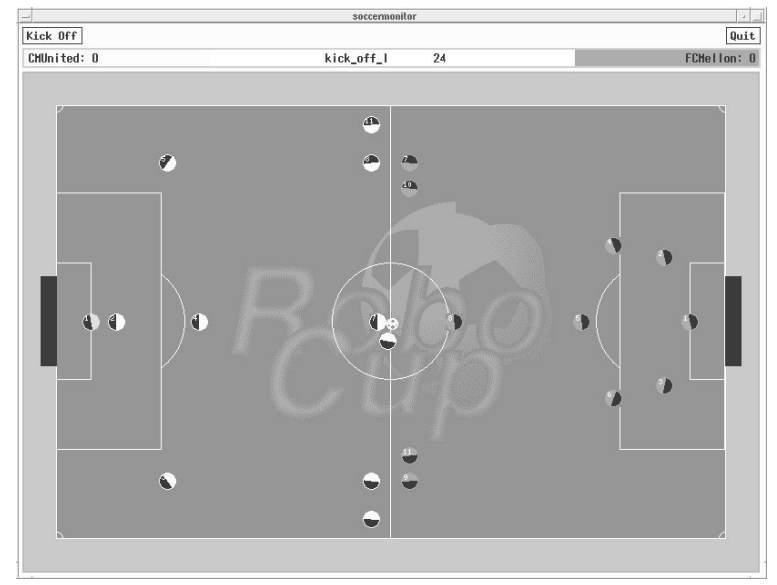

Fig. 1. The soccer server display.

The simulator also includes a referee, which enforces the rules of the game. It indicates changes in play mode, such as when the ball goes out of bounds, when 
a goal is scored, or when the game ends. It also enforces the offsides rule. Like in real soccer, a player is offsides if it is in the opponent's half of the field and closer to the opponent's goal line (the line along which the goal is located) than all or all but one of the opponent players when the ball is passed to it. The crucial moment for an offsides call is when the ball is kicked, not when it is received: a player can be behind all of the opponent defenders when it receives a pass, but not when a teammate kicks the ball towards it. ${ }^{1}$ The offsides rule, which typically plays an important role in shaping soccer strategies, is not enforced in any of the other RoboCup leagues.

The simulator, acting as a server, provides a domain and supports users who wish to build their own agents (also referred to as clients or players). Client programs connect to the server via UDP sockets, each controlling a single player. The soccer server simulates the movements of all of the objects in the world, while each client acts as the brain of one player, sending movement commands to the server. The server causes the player being controlled by the client to execute the movement commands and sends sensory information from that player's perspective back to the client.

When a game is to be played, two teams of 11 independently controlled clients connect to the server. Thus, it is a fully distributed, multiagent domain with both teammates and adversaries. The simulation league is the only RoboCup league that uses teams of 11 players as in real soccer.

The sensory information sent from the server to each client provides only a partial world view at any given moment. Each player can only "see" objects within a limited angle of the direction it is facing, and both the accuracy and description-detail of seen objects degrades with distance. In particular, sensory information is partial and noisy. Both agent action and object movement are noisy as well.

Another of the real-world complexities embraced by the soccer server is asynchronous sensing and acting. Whereas most AI simulators use synchronous sensing and acting: an agent senses the world, acts, senses the result, acts again, and so on. In this paradigm, sensations trigger actions. On the other hand, both people and complex robotic systems have independent sensing and acting rates. Sensory information arrives via different sensors at different rates, often unpredictably (e.g. sound). Meanwhile, multiple actions may be possible in between sensations or multiple sensations may arrive between action opportunities.

The soccer server communication paradigm models a crowded, low-bandwidth environment. All 22 agents use a single, unreliable communication channel. When an agent "speaks," nearby agents on both teams can hear the message. Agents have a limited communication range and a limited communication capacity, both in terms of message length and frequency.

Another limited resource of the agents is stamina. The more the agents run, the more tired they get, so that future running is less effective. Stamina has both a renewable component, that replenishes if the agents stands still, and an unrenewable component that can degrade over the course of the game.

\footnotetext{
${ }^{1}$ The soccer server operationalizes the offsides rule making it an objective call.
} 
Finally, soccer server agents, like their robotic counterparts, must act in real time. The simulator uses a discrete action model, collecting player actions over the course of a 100 msec cycle, but only executes them and updates the world at the end of the cycle. If a client sends more than one movement command in a simulator cycle, the server chooses one randomly for execution. Thus, it is in each client's interest to try to send at most one movement command each simulator cycle. On the other hand, if a client sends no movement commands during a simulator cycle, it loses the opportunity to act during that cycle, which can be a significant disadvantage in a real-time adversarial domain: while the agent remains idle, opponents may gain an advantage.

In summary, the RoboCup soccer server is a fully distributed, multiagent domain with both teammates and adversaries. There is hidden state, meaning that each agent has only a partial world view at any given moment. The agents also have noisy sensors and actuators, meaning that they do not perceive the world exactly as it is, nor can they affect the world exactly as intended. In addition, the perception and action cycles are asynchronous, prohibiting the traditional AI paradigm of using perceptual input to trigger actions. Communication opportunities are limited; the agents have limited stamina and the agents must make their decisions in real-time. These italicized domain characteristics combine to make the RoboCup soccer server a realistic and challenging domain.

\section{$2.2 \quad$ Research Challenges}

Research directions in the RoboCup simulation league are quite varied, as is evident from the articles in this book that are based on simulation research. This section presents a small sample of these directions.

The RoboCup synthetic agent challenge [8] identifies three major simulationbased challenges as being:

1. machine learning in a multiagent, collaborative and adversarial environment,

2. multiagent architectures, enabling real-time multiagent planning and decisionmaking, in service of teamwork, and

3. opponent modeling

Much past research has been devoted to these topics as reflected in this and the past RoboCup books [6,3].

Several other challenges are suggested by the characteristics of the soccer server presented above. For example, asynchronous sensing and acting, especially when the sensing can happen at unpredictable intervals, is a very challenging paradigm for agents to handle. Agents must balance the need to act regularly and as quickly as possible with the need to gather information about the environment. For example, the runner-up of the 1999 competition, magmaFreiburg, used an action-selection method based on extended behavior networks that generated decisions very quickly. This method was used primarily for times when an agent was in possession of the ball.

Some other research areas related to agent-development in the simulation league include: 
- communication in single-channel, low-bandwidth communication environments,

- social conventions, or coordination without communication,

- distributed sensing, and

- resource management.

It is interesting to note that different techniques are generally used for agent control when the agents are not in possession of the ball. Many teams use the concept of flexible formations in which agents adjust their positions based on the ball's location (e.g., [13]). Some research is focussed on using machine learning or linear programming techniques to allow agents to adapt their positioning based on the locations of the opponent players during the course of a game (e.g., [1]).

In addition to soccer-playing agent development, the soccer server has been used as a substrate for 3-dimensional visualization, real-time natural language commentary, and education research.

Figure 1 shows the 2-dimensional visualization tool that is included in the soccer server software. SPACE [12] converts the 2-dimensional image into a 3dimensional image, changing camera angle and rendering images in real time.

Another research challenge being addressed within the soccer server is producing natural language commentary of games as they proceed. Researchers aim to provide both low-level descriptions of the action, for example announcing which team is in possession of the ball, and high-level analysis of the play, for example commenting on the team strategies being used by the different teams. Commentator systems for the soccer server include ROCCO [2], MIKE [10], and Byrne [4].

Robotic soccer has also been used as the basis for education research. A survey of RoboCup-97 participants indicates that the majority of participants were students motivated principally by the research opportunities provided by the domain [14]. There has also been an undergraduate AI programming course based on teaching students to create robotic soccer-playing agents in the soccer server [5].

\subsection{The RoboCup-99 Tournament}

As with RoboCup-97 and RoboCup-98, teams were divided into leagues. In the preliminary round, teams played within leagues in a round-robin fashion, and that was followed by a double-elimination round (where a team has to lose twice to be eliminated) to determine the first three teams. Many of the games were extremely exciting, leading up to the final — watched by several hundred peoplein which CMUNiTED-99 defeated Magma FreibURG by a score of 4-0.

With respect to the competition entrants themselves, there is concrete evidence that the overall level improved significantly over the previous year. The defending champion team, the CMUNITED-98 simulator team was entered in the competition. Its code was left unaltered from that used at RoboCup-98 except for minor changes necessary to update from version 4 to version 5 of the soccer simulator. In 1998, this team won all of its matches and suffered no goals 
against. However, this year, after advancing to the elimination round, it won only one game before being eliminated.

An interesting improvement to the soccer simulator in 1999 was the addition of an on-line coach. Each team was permitted to use a single agent with an overhead view of the field that could communicate with all teammates whenever play was stopped (i.e. the ball was out of bounds). At least one team took advantage of this feature to have the coach give advice to the team regarding the overall formation of the team, which could range from offensive to defensive, and "narrow" (concentrated near the middle of the field) to wide.

Building on the success of the 1999 tournament, the RoboCup-2000 simulator tournament has even more entrants and promises to be another exciting event spawning new research approaches and successes.

\section{F-180: Small-Size Robot League}

The F-180, or "small-size" RoboCup league, features up to five robots on each team in matches on a field the size of a ping-pong table. Each robot can extend up to $18 \mathrm{~cm}$ along any diagonal and occupy up to $180 \mathrm{~cm}^{2}$ of the pitch. Color markers on the field, the robots and the ball help computerized vision systems locate important objects in the game. The robots are often controlled remotely by a separate computer that processes an an image of the field provided by an overhead camera. A couple of teams, and probably more in the future, included on-board vision.

In this section we will review the characteristics of the F-180 league, the research challenges facing teams competing in the league, and recent research contributions by some of the competing teams.

\subsection{Characteristics of the F-180 League}

The playing surface consists of a green ping-pong table enclosed by white walls. One goal area is painted yellow, while the other is painted blue - these colors help robots with onboard vision find the goals. In 2000, the league is moving to a carpeted surface of the same dimensions.

To help competitors locate their opponents, each robot carries a single colored ping-pong ball provided by the RoboCup organization. The marker is located at the geometric center of the robot as viewed from above. One team is fitted with yellow markers, while the other is equipped with blue ones. At RoboCup-99, the team carrying yellow markers attacks the blue goal and blue team attacks the yellow goal. In addition, the robots may be colored with additional markers to help computer controllers locate and orient them.

\subsection{Research and Engineering Challenges}

The core issues faced by RoboCup F-180 researchers include the construction of the robots, development of individual robot skills, reliability in dynamic, uncer- 
tain and adversarial environments, and importantly, cooperative team coordination. In the F-180 league these capabilities depend significantly on underlying engineering like reliable real-time vision and high-performance feedback control of small robots.

Competitors in the F-180 league must address most of the challenges faced by teams engaged in the simulator competition (e.g., cooperation, localization, strategy and tactics). Additionally, however, vision systems for tracking the robots must be developed and hardware to execute the control commands (the robots themselves) must be built. In terms of the autonomy required of robots, the F-180 league lies somewhere between the F-2000 league and simulation. The difficulties of locating the ball, other robots, and opponents is reduced in comparison with the F-2000 league because an overhead camera is allowed. However, the technical challenges of real-time visual tracking, feedback control and team play remain.

The visual tracking problem for the small-size league can actually be seen as more difficult in some ways than for robots in the middle-size league. The computer responsible for processing images from an overhead camera must be able to simultaneously estimate the locations and velocities of 10 robots and the ball. In some cases these robots move as fast as $2 \mathrm{~m} / \mathrm{s}$, while the ball has been recorded at speeds of $6 \mathrm{~m} / \mathrm{s}$. This vision task is being addressed using a wide range of technologies including: specialized Digital Signal Processing (DSP) hardware, commercial color tracking systems, and fast PCs equipped with commodity color capture hardware but programmed with highly-optimized image processing software. Pioneered by the CMUnited-97 and CMUnited-98 vision processing algorithms, several teams currently predict the future trajectory of the ball, and use this prediction to intersect the ball. At RoboCup-99, most of the top teams, in particular the three small robots of the RobotIS team, impressively intersected and controlled rather fast moving balls.

In addition to addressing vision and position control issues, robots must be able to manipulate the ball. Skills such as dribbling, passing and shooting are critical to successful play. It is also important for robots to be able to remove the ball from along the walls. Many robots are also equipped with devices for kicking the ball. Determining when to activate the kicker can be a tricky tactical decision.

RoboCup-99 saw a substantial increase in the mechanical capabilities of robots. In past years, a majority of the teams mainly focused with vision processing, obstacle avoidance, and cooperative behaviors. This year several teams seemed to have focused on player skills.

One of the most interesting developments concerned ball kicking technologies. At RoboCup-98, only a few teams had their own kicking devices, in particular the winning CMUnited-98 team. However the devices used in 1998 did not seem to be significantly effective. At RoboCup-99 nearly half of the participating teams utilized some sort of kicking device. One team (the FU-Fighters from Berlin) was remarkably able to propel the ball so fast that observers could barely track it (see Figure 2). 


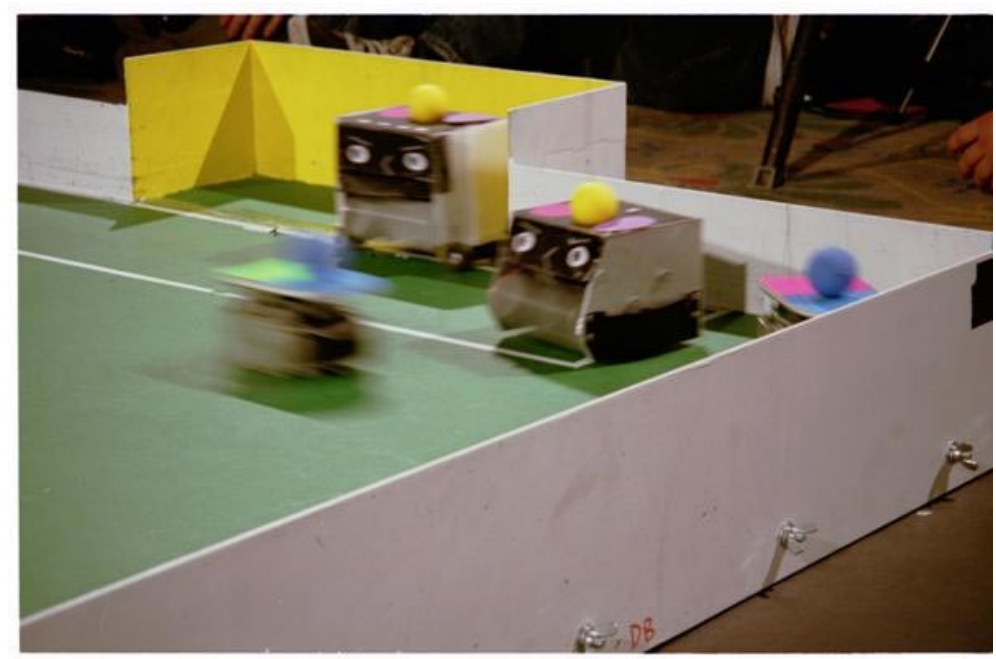

Fig. 2. The FuFighters robots with their kicking device.

Another interesting development was a new spinning technique for removing stuck balls from along the wall or in corners. The RoboCup-99 winning Big Red team demonstrated this early in the tournament and several others were able to also adopt the spinning behavior.

\subsection{The RoboCup-99 Tournament}

Participation in the Small-Size league RoboCup soccer continues to grow at a remarkable pace. Competitions in 1997 and 1998 included five and eleven competitors respectively. In anticipation of even more participants in 1999, the league instituted qualification rules to limit the field to a manageable number and to ensure groups did not travel to Stockholm with no reasonable hope of competing. In order to qualify, each team had to submit a video tape by April demonstrating at least one robot able to move the ball across the field and score (this may sound easy, but it is in fact a very challenging problem). Eighteen teams from around the world qualified for the third annual competition. The group included teams from Australia, Belgium, France, Germany, Japan, Korea, New Zealand, Portugal, Singapore, Spain, and the USA.

For the round-robin phase, the 18 teams were split into four groups of four or five teams each. In an effort to ensure equally competitive divisions each group included one of the top four finishers from RoboCup-98 and one or two new competitors. Also, no two teams from the same country were placed in the same group. During the round-robin phase, each team in each group played each of the other teams in its group. Group standings were determined by awarding three points to a team for each game it won and one point for each tie. The top two teams from each division progressed to the single elimination tournament. 
Because of the large number of teams, four separate fields were required for the round-robin. Scheduling the round-robin tournament was challenging because teams sometimes ran into technical problems and asked for delays. The task was complicated by the fact that many teams used the same frequencies for controlling their robots, and therefore could not play at the same time. Games were played twelve to thirteen hours a day for two days; there were almost always two games running concurrently.

At the end of the round-robin, the top two teams from each group (eight teams in all) took a day to move to the central conference location for the finals. This move was a bit more difficult than had been anticipated. Fortunately, all of the teams were able to adapt to the new lighting conditions and slightly cramped environment. The new location boosted attendance and crowd participation significantly. The quarter finals, semi-finals and final match conducted over the next two days in standing-room-only conditions. The final game resulted in the runner-up FuFighters and the winning BigRed team (see Figure 3).

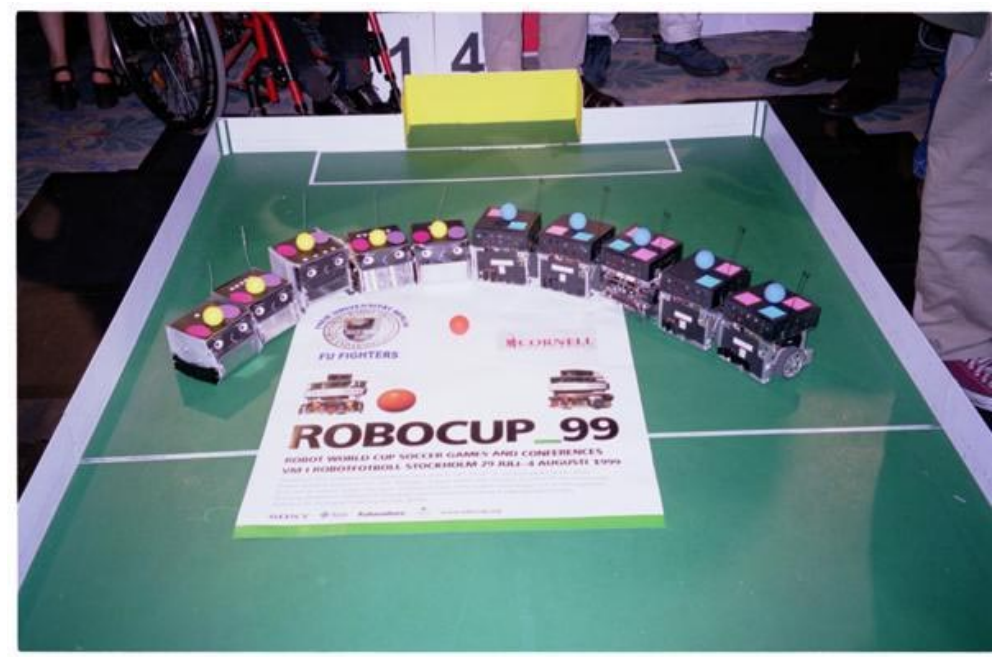

Fig. 3. The FuFighters runner-up team and the BigRed champion team.

\subsection{Evolution of the Rules}

Rules for F-180 league robotic soccer continue to evolve. Of course the long term vision for RoboCup is participation in the real human World Cup, so our robots must eventually be capable of play according to FIFA (the World Cup rules-making body) regulations. For now, however, we adjust FIFA's rules to accommodate our robots. Examples of RoboCup adjustments to the rules include special markings to help with vision issues and walls around the pitch to keep the ball from departing the playing surface. 
One detail of the rules is particularly interesting from a philosophical point of view. In real soccer, yellow cards are assigned to individual players who commit serious fouls; this approach is also used at RoboCup. In both FIFA and RoboCup soccer, when an individual receives two yellow cards, he/she/it is ejected from the game and cannot be replaced (reducing the number of players on the field). When a star human player receives a yellow card, the team's coach is faced with an important decision: should the star player be kept in the game and bear the risk that of receiving another yellow card, or should the star be replaced with a substitute? The situation is completely different for robot teams. Competitors often have a number of identical "spare" robots that can be immediately substituted for a penalized player — several teams followed this strategy.

This kind of substitution was perfectly legal, but seems to violate the spirit of the rule which is intended to punish the "offender." But which is the offender, the robot hardware or the software? Should the physical hardware be tagged with the yellow card, or should it apply to the software controlling it? This issue has been addressed in 2000 by changing the manner in which yellow cards are tracked: now they are tracked against the team as a whole. Every time two yellow cards are assigned, one player must be removed from the field.

\subsection{Lessons Learned and Current State of the League}

Probably the most frequent difficulty faced by teams in the F-180 league concerns fast vision processing. Even though many teams' vision systems work perfectly in the lab, after being re-located half-way around the world it is often a great challenge to re-calibrate them in a new environment. Problems are caused by the specific height of the camera, the variable intensity of field lighting, and the spectrum of illumination provided by the lights. Still another source of vision problems concerned the colored markers worn by opponent teams. These difficulties highlight the importance of robust vision for robots - this is a substantial challenge in nearly all domains of robotics research.

Another important lesson from successful teams is that as much effort must be applied to software development as is devoted to hardware design. It is common to see beautiful hardware designs with poor or very slow control algorithms. The winners and other top-ranked teams in previous RoboCups and also at RoboCup-99 clearly balanced their development effort between hardware and software.

The league is in great shape. It continues to draw more researchers each year. We expect about 20 teams to compete in RoboCup-2000 at Melbourne. The rules continue to be dynamic, and to reflect the research interests and directions of the participants. Two significant changes for the future include a shift to a more realistic carpet surface, and a switch to angled walls that allow the ball to leave the field more easily. In the future we hope to remove walls altogether. RoboCup in the 2000s promises to be even more exciting than in the last millennium! 


\section{F-2000: Middle-Size Robot League}

The RoboCup F-2000 League, commonly known as middle-size robot league, poses a unique combination of research problems, which has drawn the attention of well over 30 research groups world-wide. In this section, we briefly describe the fundamental characteristics of the league and discuss its major differences to other leagues. Then we present some typical research problems and the solutions developed by F-2000 teams. This overview is selective, while this book provides a rather complete survey of the research performed in F-2000 league. In particular, the references to the technical contributions from the different teams can be found as chapters in this book. We conclude with a summary of the RoboCup99 middle-size league tournament in Stockholm and a few observations on team performance and the current state of the league.

\subsection{Characteristics of F-2000 League}

Two major factors influence the design of teams and robotic soccer players for middle-size robot league: (a) the playing environment, in particular, the field, and (b) constraints imposed on robot design.

The playing environment is carefully designed such that the perceptual and motory problems to be solved are reasonably simple, but still challenging enough to ignite interesting and serious research efforts. The field size is variable within certain bounds; in Stockholm, the field size was $9 m \times 5 m$. The goals do not have nets, but colored walls in the back and on the sides (yellow/blue). The field is surrounded by white walls $(50 \mathrm{~cm}$ height) that carry a few extra markings (squared black markers of $10 \mathrm{~cm}$ size plus black-and-white logos of sponsors in large letters). A special corner design is used and marked with two green lines. The goal lines, goal area, center line and center circle are all marked with white lines. The ball is dark orange. Illumination of the field is constrained to be within 500 and 1500 lux. Matches are played with teams of four robots, including the goalie.

The robots must have a black body and carry color tags for team identification (light blue/magenta). Quite elaborate constraints exist for robot size, weight, and shape; roughly, a robot body may have about $50 \mathrm{~cm}$ diameter and be up to $80 \mathrm{~cm}$ high, must weigh less than $80 \mathrm{~kg}$, and feature no concavities large enough to take up more than one-third of the ball diameter. The robots must carry all sensors and actuators on-board; no global sensing system is allowed. Wireless communication is permitted both between robots and between robots and outside computers.

\subsection{Research and Engineering Challenges}

A general survey of RoboCup research issues can be found in [6,3]. An interesting perspective on middle-size league research issues results when compared with the simulation league. Ultimately, all major research issues in simulation league, like coordinated team play, opponent modeling, game strategy and tactics, in-game 
adaptation to opponent tactics, etc., have to be solved in middle-size league as well. However, while agent design in simulation league can build upon a set of reasonably reliable perception and action commands (e.g., it is possible to precisely and deterministically determine a player's position and orientation on the field with little computational effort), it is a non-trivial task to achieve this level of player capability with real robots. Thus, building a F-2000 robot team starts with a combined engineering and research challenge: choosing or designing appropriate robots.

A basic decision with far-reaching consequences is the solution selected to achieve mobility. A wide spectrum of alternative solutions developed in classical robotics is available; for example, omnidirectional drive systems allow to design very agile robots, but can be quite complex to control. Differential drive systems are much easier to control, but often require more complex movement maneuvers during play. Another mechanical engineering problem is the design of mechanisms for handling the ball. Because ready-to-use solutions were hardly available, this problem has led to a wide range of different approaches and interesting new designs.

Although a number of small, commercial robot platforms have become available over the past few years (e.g., the Pioneer series by Activmedia and the Scout robots by Nomadics, both of which use differential drive systems), almost none of them can be considered a complete robotic soccer player. Typical items teams found necessary to add include mechanical kicking devices, additional sensors like bumpers, compasses, laser scanners, unidirectional and omnidirectional cameras, and additional computing power (notebook computers, embedded computers, DSP boards). Because off-the-shelf soccer robots are not available, a substantial number of teams decided to build their own robots. In either case, the time and effort needed to design and construct (or acquire) all necessary components and to integrate them into a reliably working soccer robot is often grossly under-estimated and under-valued. At the very least, this kind of system's integration work is a great educational experience for students.

Once a functional physical robot is available, a number of basic perceptual and behavioral problems must be solved. Perception and action commands are needed for the following functions:

- Detection and tracking of the ball.

- Detection and tracking of the goals, corners, lines, and other landmark features of the field.

- Detection and recognition of teammates and opponents.

- Kicking and passing the ball.

- Dribbling the ball.

- Goalie.

All this basic functionality, which has proven to be very hard to obtain in a robust and reliable manner, is already available for designers of soccer players in the simulation league. Also, this functionality is achieved in the small-size robot league through an outside camera mounted above the field. The images to 
be processed in the small-size league are characterized by comparatively stable lighting conditions (lighting comes from the same direction as the camera) and little optical flow. The goals and the walls surrounding the field remain stable, and only the position of the ball and the players change. Vision processing in the F-180 league is however still very challenging due to the typically very high speed of both robots and the ball. The small-size researchers have developed fast vision processing routines (up to $60 \mathrm{~Hz}$ frame rate) to detect and track in real time up to the eleven fast moving objects on the field.

The situation is completely different in the F-2000 league, where the camera is near the floor (lighting direction is almost perpendicular to camera direction) and the lighting situation is far less stable. As a consequence, for example cameras see the ball as an ensemble of three differently colored regions: a red portion in the middle, a white portion at the top (reflection of lighting for the field), and a black portion at the bottom (the shadowed lower part of the ball and its own shadow). Also, the camera is actively moved through the environment, resulting in images where everything constantly changes, especially the distances to objects and landmark features to be recognized. Usually, only a small portion of the environment is visible at any given time; 30 to 120 degree visual angle for unidirectional cameras and a visual field of about $2 \mathrm{~m}$ around the robot for omnidirectional cameras are typical. Detecting and tracking the relevant objects and landmark features requires robust and reliable techniques for color-based and texture-based image segmentation, line detection, and the combination of color, shape, and texture feature for object recognition and tracking. In summary, the middle-size research platform offers a very challenging setting regarding the perceptual situation of robotic soccer players.

On the behavioral side, hand-crafting robust and reliable action commands for kicking or passing the ball into a certain direction (and possibly, with varying strength) as well as moving with ball such that the robot maintains control over the ball (necessary for dribbles) often require substantial programming and tuning effort. An interesting research challenge is to develop tools for programming and debugging such behaviors modules and to apply learning techniques to this problem.

Due to these constraints, constructing the basic functionalities listed above proves to be a very hard problem. The quality of the solutions achieved for these problems usually directly influences the performance level for the next level of functionality, which includes

- world modeling,

- self-localization,

- obstacle detection and avoidance of or recovery from collisions, and

- behavior engineering, especially behaviors for finding the ball, dribbling the ball, passing to teammates, shooting a goal, performing a penalty kick, etc.

World modeling and self-localization in RoboCup are interesting because the environment is highly dynamic, currently containing nine almost constantly moving objects. Several other state-of-the-art mobile robot applications, where 
robots are also in highly dynamic environments like museums, treat all dynamic objects as obstacles which have no direct relevance to the task at hand. On the contrary, soccer robots cannot take this simplified view. The development of probabilistic representations for highly dynamic environments, like robotic soccer, is a challenging and still open research problem. Accordingly, adapting existing techniques for self-localization to work with such representations is required. For teams following an approach with some kind of central team coordinator (often by an outside computer) the integration of partial (and possibly inconsistent) world models provided by individual players is another research topic, which is now also investigated outside of the RoboCup community.

Obstacle detection and avoidance or recovery from collisions is a difficult problem in RoboCup, because of the intricate rulings on charging and foul play. Although soccer is a sport where physical contact is not always avoidable, there is mutual understanding in the community that pure robot strengths should not be a "winning factor"; charging fouls have drastic consequences, up to exclusion from the tournament. On the other hand, robots being overly cautious to avoid physical contact may give way to their opponents too easily. Thus, we encounter a very difficult situation assessment and classification problem.

The effort of hand-crafting more complex behaviors, like dribbling the ball or performing a penalty kick, is even higher than those mentioned before. Thus, there is a large need for behavior engineering tools, and for techniques applying learning and on-line adaptation to the behavior engineering and action selection problems.

Above the level described so far, the research challenges are quite similar to those in the simulation league. Some research groups are actually active in more than one league (e.g, CMU, Ulm, Italy, Portugal) and hope to apply results regarding strategic and tactical play from their simulation team to the robot team in the near future. An interesting design issue is that while each robot of most teams showed the same technical characteristics of its playmates, or at maximum differentiated with respect to the goalkeeper, the ART team was forced to put playing together all kinds of robots. The necessity of forming a team with robots, having different mechanics, different hardware, different software architectures, and different sensors, led to the development of a specific ability of organizing a heterogeneous multi-robot system where it would be possible to replace, at any time during the game, a specific robot with a different one. This ability was achieved by the dynamic assignment of different roles through the evaluation of some utility functions (team Italy).

For the sake of completeness, it should be noted that, in the F-2000 league, modeling player stamina is usually not investigated, while it is a considerable problem for many simulation teams. Also, F-2000 players may use considerable bandwidth in communication both between themselves and with outside computers, which allows teams to apply more centralized team architectures (team Osaka). Constraints on communication are stricter in simulation, and all soccer agents must have a high degree of autonomy, while in F-2000 only few teams follow this idea. 


\subsection{Examples of Engineering and Research Results}

Substantial effort has been spent in most teams on actually designing robotic soccer players. The Australian team RMIT Raiders won the RoboCup-97 technical innovation award for their omnidirectional drive system design that is modeled after a computer mouse. The Japanese team Uttori United, a joint effort by three research labs, developed another omnidirectional drive design in 97 and 98 that uses four so-called Swedish wheels (wheels with free rollers at the rim) arranged in a rectangular setup. Such drive designs are usually quite complex to control, but the Uttori design applies three actuators and an elaborate transmission mechanism to decouple the various degrees of freedom (DoFs): each actuator contributes only to its corresponding DoF. In RoboCup-99, the team from Sharif University of Technology (Iran) presented robots with 4 DoFs mobile base consisting of two independently steered and actuated wheels plus additional castor wheels. This design provided excellent mobility and speed that contributed much to the overall success of the team in RoboCup-99.

A wide range of different kicking devices have been developed. Several teams use electrically activated pneumatic cylinders as actuators for kicking in order to get sufficient kicking power (e.g., teams Italy, Matto, and Ulm). In particular, Bart and Homer (the two robots designed at the University of Padua that played with the ART team), were equipped with a flexible directional kicker that had a left and right side able to slide one to each other, in order to acquire high flexibility and accuracy. Some teams built complete robots from scratch using standard industrial equipment as components where possible (e.g., teams GMD, Italy, Matto, and Ulm). An interesting method for easy integration of microcontroller-driven sensing and actuating devices is based on the CAN bus that allows to connect up to 64 devices on a single 1 Mbit bus (team Ulm).

Aside of the mechanical design and engineering questions, the research efforts of teams in the middle-size robot league clearly indicate several focal points: vision, localization, and behavior engineering.

In the vision area, methods for fast color image segmentation have been developed and continue under research. Several teams use omnidirectional cameras and develop methods for processing the images, in particular for self-localization and object recognition (e.g., teams Italy, Tübingen, Osaka, Matto, and Portugal). The Italian team developed special mirror designs in order to extend the field of view in general and to combine a view of the local surrounding with a more global view of localization-relevant parts of the environment (walls and goals).

A scan-matching approach to self-localization has been the key to the success of the CS Freiburg team in RoboCup-98. Although the use of laser scanners on every robot means significantly more weight and power consumption, having a specialized sensing system for localization tends to make camera control and vision processing simpler. In fact, visually tracking the ball and opponents and trying to find localization-relevant visual features of the environment (corners, walls, goals) at the same time (or interleaved) often causes conflicts in view direction. 
Another interesting direction in localization investigates approaches to visionbased self-localization. Iocchi et al. use a Hough transform for localization purposes in the team from Italy. Ritter et al. extended Monte-Carlo-Localization, a model-based method developed mainly for use with laser range finders, to work with features extracted from camera images. Other effective extensions of MCL were achieved within by Carnegie Mellon within the Sony legged robot league. Compared to standard MCL, vision-based features in RoboCup are very few and can be detected far less frequently and reliable than laser scan points, but recent results prove that the extended MCL framework is functional even under these restrictive constraints. The Agilo RoboCuppers from Munich and the team Attempto from Tübingen both use model-matching methods for localization.

Perceiving the relevant objects and knowing where the robot is are important prerequisites for generating successful soccer playing behaviors. The team from GMD uses a behavior-based approach, called dual dynamics, and presented tools for designing behaviors for a single player without giving particular attention to cooperative play. The Italian team also developed tools for designing behaviors effectively.

A couple of teams already started to seriously investigate methods for generating cooperative playing skills. In particular, Bart and Homer, from the University of Padua, achieved the cooperative ability of "exchanging the ball" between two players (a kind of action less complex than "passing a ball") through the implementation of efficient collision avoidance algorithms activated in the framework of the dynamic role assignment used by ART. The team from Osaka University has already significant experience in methods for generating cooperative playing skills and in applying reinforcement learning techniques to this problem.

\subsection{The RoboCup-99 Tournament}

The middle-size (F-2000) RoboCup-99 tournament went very smoothly. Twenty teams participated and played a total of 62 games, giving all teams ample opportunity to gain practical playing experience. The new rule structure for the middle-size robot league, which is based upon the official FIFA rules, proved to be quite successful and helped to focus on real research issues instead of rule discussions.

Just as in real soccer, the games were very exciting and unpredictable (see Figure 4). Several teams, which performed well in the past and have already won a cup, suffered unexpected losses, often against strong newcomers like SHARIFCE (Sharif University of Technology, Iran), AlPhA ++ (Ngee Ann Polytechnic, Singapore), and WISELY (Singapore Polytechnic, Singapore), and did not survive the preliminary rounds. The 20 participants were distributed into three groups, which came up with eight finalist teams.

The most struggled game was one of the semi-finals, when the Italian Team (ART) won over the then-undefeated champion of RoboCup-98, CS FREIBURG, in a match that required one penalty kick round, and two technical challenge rounds to come up with a decision. This game showed the real achievement of 


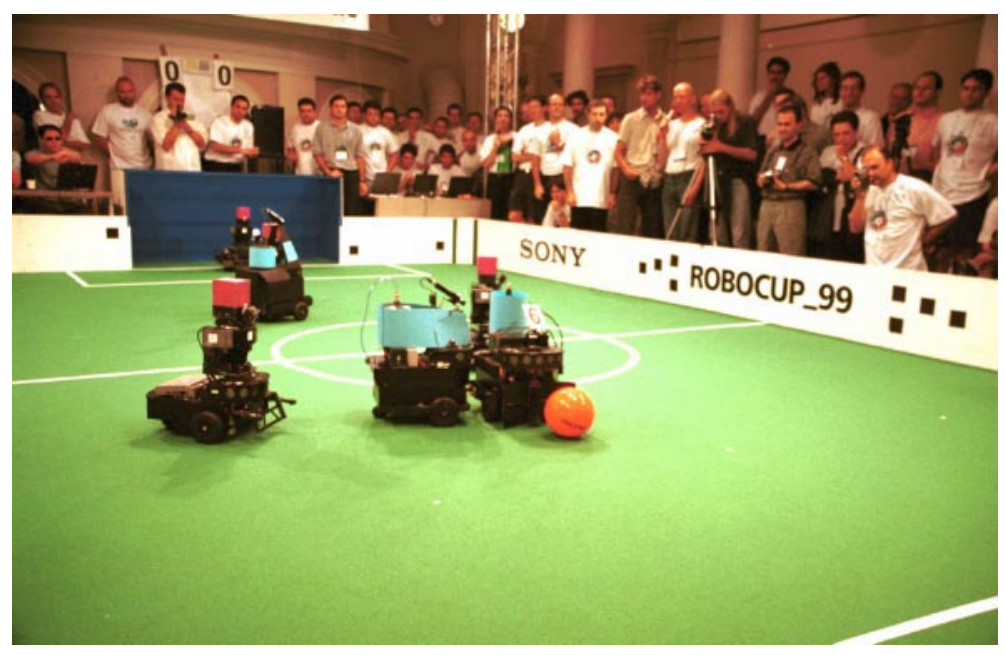

Fig. 4. A view of a middle-size game.

the third RoboCup Physical Agent Challenge (the explicit passing of the ball between two players), when a German robot, which was controlling the ball near the opposite goal, waited for its playmate to reach a good position and then passed the ball to it. However, high-level reasoning capabilities of CS FREIBURG robots in general were not sufficient by themselves to defeat ART robots. Based on a set of reactive behaviors, especially when Bart and Homer played together, the ART team was able to generate emergent cooperative abilities.

In a very exciting final game, a crowd of several hundred spectators watched how the team from Sharif University, SHARIF CE, defeated the ART team by $3: 1$. Also this game showed the achievement of a difficult challenge, when an Iranian robot was able to perform a perfect dribbling and scored a goal in a few seconds from the start of the game.

\subsection{Lessons Learned and Current State of the League}

One thing one can learn from the tournament is that hardware alone does not buy success. Several teams, in spite of a sophisticated robot design with advanced tools like both directional and omni-directional cameras, cognachrome vision systems, laser scanning, expensive on-board laptops, did not always perform better than less high-tech teams. This fact proves that complex hardware requires substantial time to develop adequate software that can actually exploit the hardware features. On the other hand, hardware innovations can also be the foundation for success. The 1999 champion, SHARIF CE, benefited substantially from the agility of their robots, which arose from a combination of clever drive design and speed. Overall, systems that manage to exhibit relatively few behaviors, in a very robust and reliable manner, seemed to be more successful than more complex, but less reliable, systems. 
Overall, the league is in good shape. Worldwide, well over 30 teams are working on building and improving a middle-size robot team. Provided that the rules and the playing field remain reasonably stable for the near future, we expect significantly enhanced vision capabilities, much improved ball control, smoother individual behaviors, and increasingly more cooperative playing behaviors. It will be a lot of fun to watch the RoboCup-2000 and RoboCup-2001 tournaments!

\section{Sony Legged Robot League}

Sony Legged Robot League is a new official RoboCup league since RoboCup-99. Four-legged autonomous robots compete in three-on-three soccer matches. The robot platform used is almost the same as the Sony AIBO entertainment robot that was introduced into a general consumer's market last July. 5000 sets were immediately sold, namely 3000 in Japan in 20 minutes, and 2000 in the US in four days.

The robot platform used in this league is modified from the commercial product version so that the RoboCup participating teams can develop their own programs to control the robots. Since hardware modifications to the robots are not allowed, the games are decided by who has developed the best software. The robots are equipped with a CCD vision camera.

The playing field is carpeted and slightly wider and larger than the smallsize field. In order for a robot to be able to localize itself, the important game items, namely the ball and the goals, are painted in different colors. The ball is orange and the goals are yellow and blue. The field also includes six colored distinguishable landmark poles at the corners and the middle of the field. Robots can use the colored landmarks to localize themselves in the field.

Each team has three players, and a game consists of two 10min halves with a $10 \mathrm{~min}$ break. If the game is a draw at the end of the 20 minutes, penalty kicks are carried out. There is a penalty area where only one robot can defend the goal. A referee can pick up and replace robots to other locations, if multiple robots are entangled usually while competing for the ball.

\subsection{Research Issues}

Vision: Since the robots easily lose sight of the ball due to occlusion by other robots and due to the limited visual angle of their camera, they need to effectively search for the ball. Research teams need to develop image processing algorithms combined with object recognition and search.

Navigation: Most teams used the four-legged walking programs provided by Sony due to the limited time available for development of new walking algorithms and/or because they preferred to focus on the tactics of the game. A few teams developed their own walking programs, for instance LRP developed a stable and robust walking program, and Osaka developed a trot walking to increase the speed of walking. The former could show the good performance during matches while the latter did not seem consistently robust although the speed itself was better than the former. 
Playing Skills: Since the motor torque at each joint of the leg is not very powerful, kicking is not actually very effective, and pushing showed to be sufficient. Most teams used simple pushing as their shooting behavior. One exception was performed by the team ARAIBO (U. of Tokyo), as they used a heading shoot having the robots fall forward and performing interestingly.

Localization: Self-localization is one of the most important issues as the robots need to know where they are on the field. The teams attempted to use the colored landmarks for localization based on triangulation. CM-Trio-99 (Carnegie Mellon Univ.) introduced a new algorithm based on probabilistic sampling that allowed the robots to effectively process poorly modeled robot movement and unexpected errors, such as the change of robot location by the referees. The team from Osaka used the landmarks for task accomplishment. Based on an information criterium, the robot decides if more observation is necessary to determine the optimal action. CM-Trio-99 also introduced multi-fidelity behaviors that degrade and upgrade gracefully with different localization knowledge. The winning team from LRP, France mainly used the goals for localization moving fast and successfully towards the offensive goal.

Teamwork: Each player is marked with the team color, namely red and dark blue. So far, it has shown to be rather difficult to reliably detect the other robots based on their team colored patches. Most teams achieved basic teamwork through the assignment of roles, as one goalie robot and two attackers. The Osaka played with no specific goalie. The runner-up team from UNSW and CM-Trio-99 (3rd place) demonstrated interesting goalie behaviors. The attackers from LRP were quite effective at moving towards the goal. Team$\mathrm{s}$ have not yet achieved more sophisticated cooperative behaviors, such as passing.

\subsection{The RoboCup-99 Tournament}

An initial competition, as a demonstration, was held at RoboCup-98 in Paris. The prototype "AIBO" robots were used by three teams, Osaka University BABYTigers (Japan), Carnegie Mellon University CM-Trio-98 (USA), and Laboratoire de Robotique de Paris (LRP) Les Titis PARIsiens (France). CMTrio-98 was the winner of this RoboCup-98 competition.

At RoboCup-99, in addition to the three seeded teams from RoboCup-98, namely BabyTigers-99, CM-Trio-99, and "Les 3 Mousquetaires" from LRP, there were six new teams: from Sweden (Örebro, Stockholm, Ronneby and others), Humboldt University (Germany), University of Tokyo (Japan), University of New South Wales (Australia), University of Pennsylvania (USA), and McGill University (Canada).

The teams were divided into three groups, each of which included one seeded team. After the round-robin phase, all three seeded teams advanced to the elimination phases. Although the seeded teams had to develop new algorithms and implementations for the new AIBO robots, they were probably still in advantage, as the six new entering teams had only two months to develop their 
teams. The wild card for the fourth participant in the semi-finals was decided by the RoboCup Challenge. Each team had one try to have a single robot on the field score a goal in three different unknown situations. Although none of the teams successfully performed and scored, the UNSW secured the wild card spot with their steady performance (the shortest distance between the ball and the opponent goal).

In the first semi-final, Osaka gained one goal in the first half but LRP quickly recovered and scored two goals. Osaka attacked LRP's goal many times in the second half, but their attacks were blocked by the LRP's robust defense. Since Osaka had no goalie, LRP gained two more goals in the second half. LRP's walking and image processing seem to be very robust.

The second semi-final between UNSW and CM-Trio-99 was an interesting game. The two teams had already played in the round-robin phase and CMTrio-99 had won. However, in this game, the CM-Trio-99 team encountered some unexpected problems and lost 2-1 to the UNSW team. UNSW scored two goals in the first half, the first goal by squeezing the ball into the goal behind CMTrio-99's goalie. In the second half CM-Trio-99, partially recovered, could not score more than one goal, as the UNSW goalie was notably strong.

The final between UNSW and LRP started at 1:30pm on August 4, 1999, being observed by a large crowd. The first half ended with UNSW scoring a goal into its own goal. In the second half, many attacks by LRP showed their superiority and gained three goals in spite of the nice defense by the UNSW goalie. UNSW still gained one goal through a quick attack just after the kickoff. LRP won the championship. We will have twelve teams in RoboCup-2000 in Melbourne.

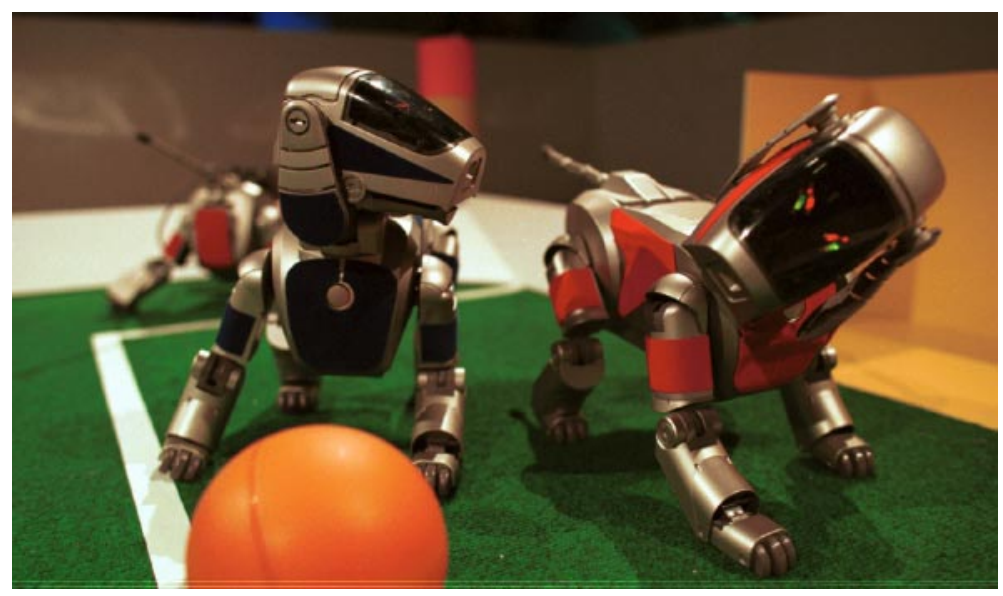

Fig. 5. Two Sony dog robots in a game. 


\section{RoboCup Jr. Exhibition}

RoboCup Jr. is an initiative where children can get hands-on experience with advanced robotic topics. It is an educational project aiming at providing an environment for children to learn general science and technology through robotics $[7$, 9]. Due to the long range goal of RoboCup aiming at having a robotic team play a real human team in 2050, it is essential that younger generations get involved in science and technology in general, and in RoboCup activities in particular.

RoboCup Jr. is designed to enhance education using the excitement of the soccer game and the sense of the technical complexity of the real world by actually programming physical entities, instead of virtual creatures.

Unlike in other RoboCup soccer leagues that are designed for top-level research institutions, RoboCup Jr. has flexible and easy to start setup with using several robot platforms, such as LEGO Mindstorms, and easy-to-program environments. Children are expected to have a hands-on experience actually building and program robots, playing games, and learning general technical lessons from their experiences.

While RoboCup Jr. is in its infancy, we are planning to enlarge this activity to have a wide variety of educational programs in a very systematic manner with solid support from education and developmental psychology research.

\subsection{Characteristics of RoboCup Jr.}

For children to easily get involved in RoboCup Jr., several commercial robot platforms and robot kits are used with specific size and configurations.

Leagues consist of competition division, such as RoboCup Jr. Soccer League, and collaborative division, such as RoboCup Jr. Performers that involves parade and dancing. Even within the RoboCup Jr. Soccer League, several levels are planned to be provided depending upon the educational needs.

The easiest level focused on assembly of robot kits to learn how to construct robots. Similarly, the focus can be placed on how to program the robots whereas the robot themselves are already provided. The second level consists of building and program a simple robot and play a game or do parade. Both aspects of craftsmanship and programming are required. Higher levels may be arranged for more advanced children and for undergraduates that are yet to get involved in the research-oriented RoboCup leagues. The numbers of robots used may vary. A simple game can be just a one on one robot game.

The standard set up for the entry-level league is to have a field of size $120 \mathrm{~cm}$ by $90 \mathrm{~cm}$ with $5 \mathrm{~cm}$ walls around. The field has gray scale from one end to the other so that a simple sensor can detect the approximate location of robots in the field along one axis. Pre-assembled LEGO Mindstorms or equivalent are provided and children program the robots. A programming environment is available so that children can program and play a game in a short time, approximately within one hour. 


\subsection{Research Challenges}

Many research challenges arise within RoboCup Jr. The first issue is to define a comprehensive system for robotics education, and science and technology education through robotics. While there are major on-going efforts in Information Technology education, there are only a few efforts made on education with robotics as the central theme. We believe that the use of robotics greatly enhances technical education due to the sense of reality involved in using real physical objects that can be programmed.

Secondly, the development of appropriate infrastructures, such as robot kits, programming environments, and educational materials, is a major challenge. Since RoboCup Jr. aims at wide-spread activities of world-wide scope, a rigid and well-designed environment is essential. This is also an important challenge from the aspect of human-computer interaction.

While RoboCup Jr. mainly targets education for children, it can be applied for science and technology literacy for the general public with non-scientific or technological background.

\subsection{RoboCup Jr. at RoboCup-99}

In RoboCup-99, Stockholm, a small experimental exhibition was carried out using one field to play with two PCs for programming. Children showed up on the day and programmed robots to play one-vs-one soccer using pre-assembled LEGO Mindstorms. Over 60 children participated over 2 days of exhibitions and a large number of games were played, as well as informal tournaments. Children were very involved and participated actively (see Figure 6).

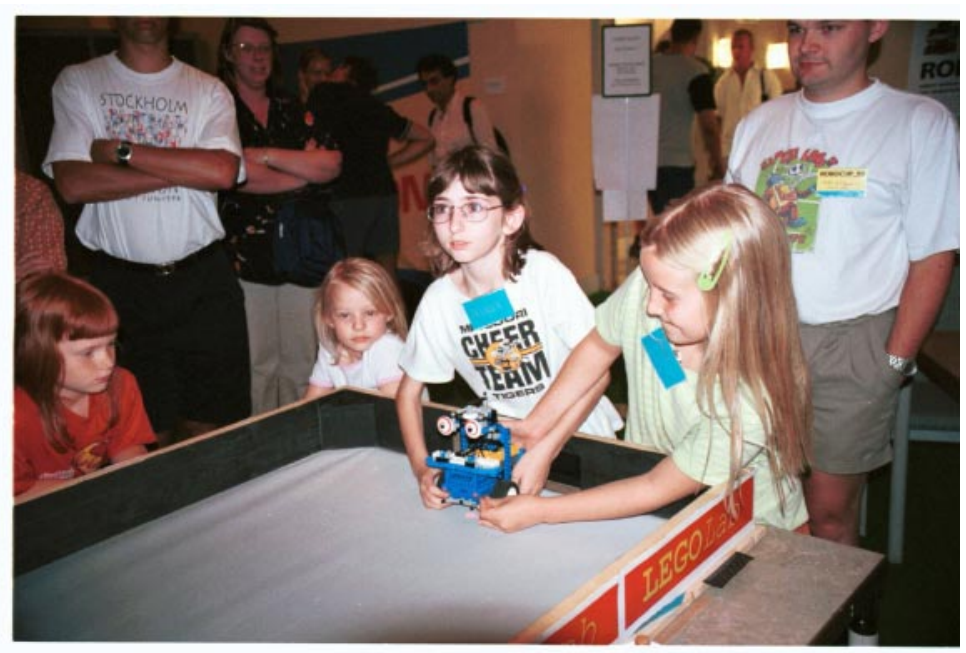

Fig. 6. Children participating at RoboCup Jr. 
RoboCup-99 included three main demonstrations of RoboCup Jr. An Israeli team showed the use of robot soccer in high school education with a penalty shooting robot developed by high school students (age approximately 16-17). Bandai showed a remotely-controlled soccer game with two robots, each with a holding and a shooting device, on each team. The LEGO Lab, as described above, developed by the University of Aarhus, arranged open sessions for children (ages 7-14) to develop their own robot soccer players and to participate in a tournament.

A few additional interesting observations can be made. First, it was confirmed that 7-9 years old children can program robots, such as the Lego Mindstorms, within 30 minutes when the appropriate environment is provided and a task is well defined. It is less feasible to expect children to be able to write code in some higher-level language. Instead there was clear evidence that an easy visual programming environment showed to be of great help.

Second, it was noticed that different children approach the robot programming task in different ways. Some immediately program the robots and others cautiously delay their programming until they have observed the essence of the games. After RoboCup-99, Henrik Lund and one of his colleagues carried out RoboCup Jr. with two robots per team at the Mindfest at the MIT Media lab., and observed other emotional reactions of children.

RoboCup Jr. events are planned for the RoboCup European Championship in Amsterdam, in May 2000 and for RoboCup-2000, in Melbourne, in August.

\section{Conclusion}

RoboCup is growing and expanding in many respects. The number of participants is increasing, and so is the complexity of the organization. A new league with the Sony legged robots was officially introduced this year. The performance of all the teams in the different leagues is clearly increasing. The research contributions are getting increasingly well identified and reported. RoboCup-99 attracted the interest of many researchers, of the general public, and of the media. RoboCup-99 continues to pursue its core goal as a research environment, stimulating and generating novel approaches in artificial intelligence and robotics. The RoboCup environment provides a remarkable concrete platform for researchers interested in handling the complexities of real-world problems.

In 2000, there will be the first RoboCup European Championship in Amsterdam, in May, with a workshop, RoboCup Jr,. and competitions in the simulation, small-size, and middle-size leagues. The annual international RoboCup2000 will be held in Melbourne, Australia, in August, in connection with the Sixth Pacific Rim International Conference on Artificial Intelligence (PRICAI2000). RoboCup-2000 will include a technical workshop, competitions in all of the four leagues (simulation, small-size and middle-size robots, and Sony legged robots), RoboCup Jr., and two new demonstrations towards a RoboCup Humanoid league and a RoboCup Search and Rescue league. 


\section{Appendix A: Round-Robin Simulation League}

This appendix includes all the results from the preliminary games in the simulation leagues. The tables show the numbers of goals, the number of wins, losses, and draws $(\mathrm{W} / \mathrm{L} / \mathrm{D})$, and the rank of each team within each group. A win was counted against a team that forfeited a game.

\section{Group A}

- A1: CMUnited-99

- A2: The Ulm Sparrows

- A3: Headless Chickens III

- A4: Zeng99

- A5: Dash (forfeited the games)

\begin{tabular}{||c||c|c|c|c|c|c||}
\hline \hline & A1 & A2 & A3 & A4 & W/L/D & Rank \\
\hline \hline A1 & - & $29-0$ & $17-0$ & $11-0$ & $4 / 0 / 0$ & 1 \\
\hline A2 & $0-29$ & - & $0-12$ & $1-0$ & $2 / 2 / 0$ & 3 \\
\hline A3 & $0-17$ & $12-0$ & - & $3-0$ & $3 / 1 / 0$ & 2 \\
\hline A4 & $0-11$ & $0-1$ & $0-3$ & - & $1 / 3 / 0$ & 4 \\
\hline \hline
\end{tabular}

\section{Group B}

- B1: 11 Monkeys

- B2: Sibiu Team

- B3: FCFoo

- B4: Pardis

- B5: Gongeroos

\begin{tabular}{||c||c|c|c|c|c|c|c||}
\hline \hline & B1 & B2 & B3 & B4 & B5 & W/L/D & Rank \\
\hline \hline B1 & - & $17-0$ & $18-0$ & $2-0$ & $4-0$ & $4 / 0 / 0$ & 1 \\
\hline B2 & $0-17$ & - & $0-5$ & $6-1$ & $0-3$ & $1 / 3 / 0$ & 4 \\
\hline B3 & $0-18$ & $5-0$ & - & $2-0$ & $2-4$ & $2 / 2 / 0$ & 3 \\
\hline B4 & $0-2$ & $1-6$ & $0-2$ & - & $0-9$ & $0 / 4 / 0$ & 5 \\
\hline B5 & $0-4$ & $3-0$ & $4-2$ & $9-0$ & - & $3 / 1 / 0$ & 2 \\
\hline \hline
\end{tabular}

\section{Group C}

- C1: Mainz Rolling Brains

- C2: IALP

- C3: RoboCup Koblenz

- C4: Erika

- C5: Polytech100

\begin{tabular}{||c||c|c|c|c|c|c|c||}
\hline \hline & C1 & C2 & C3 & C4 & C5 & W/L/D & Rank \\
\hline \hline C1 & - & $17-0$ & $25-0$ & $22-0$ & $16-0$ & $4 / 0 / 0$ & 1 \\
\hline C2 & $0-17$ & - & $1-1$ & $0-8$ & $0-2$ & $0 / 3 / 1$ & 5 \\
\hline C3 & $0-25$ & $1-1$ & - & $0-0$ & $0-0$ & $0 / 1 / 3$ & 4 \\
\hline C4 & $0-22$ & $8-0$ & $0-0$ & - & $0-0$ & $1 / 1 / 2$ & 2 \\
\hline C5 & $0-16$ & $2-0$ & $0-0$ & $0-0$ & - & $1 / 1 / 2$ & 3 \\
\hline \hline
\end{tabular}




\section{Group D}

- D1: Gemini

- D2: Footux-99

- D3: UBU

- D4: KU-Sakura-2

\section{Group E}

\begin{tabular}{||l||c|c|c|c|c|c||}
\hline \hline & D1 & D2 & D3 & D4 & W/L/D & Rank \\
\hline \hline D1 & - & $12-0$ & $14-0$ & $0-0$ & $2 / 0 / 1$ & 1 \\
\hline D2 & $0-12$ & - & $5-0$ & $0-11$ & $1 / 2 / 0$ & 3 \\
\hline D3 & $0-14$ & $0-5$ & - & $0-9$ & $0 / 3 / 0$ & 4 \\
\hline D4 & $0-0$ & $11-0$ & $9-0$ & - & $2 / 0 / 1$ & 2 \\
\hline \hline
\end{tabular}

- E1: ATHumboldt-99

- E2: Paso-Team

- E3: AIACS

- E4: NITStones

- E5: magmaFreiburg

\section{Group F}

\begin{tabular}{||c||c|c|c|c|c|c|c||}
\hline \hline & E1 & E2 & E3 & E4 & E5 & W/L/D & Rank \\
\hline \hline E1 & - & $16-0$ & $18-0$ & $9-0$ & $0-9$ & $3 / 1 / 0$ & 2 \\
\hline E2 & $0-16$ & - & $0-9$ & $0-12$ & $0-11$ & $0 / 4 / 0$ & 5 \\
\hline E3 & $0-18$ & $9-0$ & - & $1-6$ & $0-19$ & $1 / 3 / 0$ & 4 \\
\hline E4 & $0-9$ & $12-0$ & $6-1$ & - & $0-20$ & $2 / 2 / 0$ & 3 \\
\hline E5 & $9-0$ & $11-0$ & $19-0$ & $20-0$ & - & $4 / 0 / 0$ & 1 \\
\hline \hline
\end{tabular}

- F1: CMUnited-98

- F2: Essex Wizards

- F3: Karlsruhe Brainstorms

- F4: Kasuga-bito III

- F5: Cyberoos

\section{Group G}

\begin{tabular}{||c||c|c|c|c|c|c|c||}
\hline \hline & F1 & F2 & F3 & F4 & F5 & W/L/D & Rank \\
\hline \hline F1 & - & $0-0$ & $1-0$ & $19-0$ & $1-0$ & $3 / 0 / 1$ & 2 \\
\hline F2 & $0-0$ & - & $1-0$ & $20-0$ & $3-0$ & $3 / 0 / 1$ & 1 \\
\hline F3 & $0-1$ & $0-1$ & - & $32-0$ & $6-2$ & $2 / 2 / 0$ & 3 \\
\hline F4 & $0-19$ & $0-20$ & $0-32$ & - & $2-0$ & $1 / 3 / 0$ & 4 \\
\hline F5 & $0-1$ & $0-3$ & $2-6$ & $0-2$ & - & $0 / 4 / 0$ & 5 \\
\hline \hline
\end{tabular}

- G1: UvA-Team

- G2: KULRoT

- G3: Smackers-99

- G4: YowAI

\begin{tabular}{||c||c|c|c|c|c|c||}
\hline \hline & G1 & G2 & G3 & G4 & W/L/D & Rank \\
\hline \hline G1 & - & $13-0$ & $3-2$ & $0-2$ & $2 / 1 / 0$ & 2 \\
\hline G2 & $0-13$ & - & $0-11$ & $0-20$ & $0 / 3 / 0$ & 4 \\
\hline G3 & $2-3$ & $11-0$ & - & $0-21$ & $1 / 2 / 0$ & 3 \\
\hline G4 & $2-0$ & $20-0$ & $21-0$ & - & $3 / 0 / 0$ & 1 \\
\hline \hline
\end{tabular}




\section{Group H}

- H1: Oulu-99

- H2: PSI Team

- H3: Rogi2 Soft Team

- H4: SP-United

- H5: Kappa-II

\begin{tabular}{||c||c|c|c|c|c|c|c||}
\hline \hline & H1 & H2 & H3 & H4 & H5 & W/L/D & Rank \\
\hline \hline H1 & - & $1-0$ & $5-1$ & $1-0$ & $0-11$ & $3 / 1 / 0$ & 2 \\
\hline H2 & $0-1$ & - & $0-5$ & $0-1$ & $0-2$ & $0 / 4 / 0$ & 5 \\
\hline H3 & $1-5$ & $5-0$ & - & $0-0$ & $0-13$ & $1 / 2 / 1$ & 4 \\
\hline H4 & $0-1$ & $1-0$ & $0-0$ & - & $0-5$ & $1 / 2 / 1$ & 3 \\
\hline H5 & $11-0$ & $2-0$ & $13-0$ & $5-0$ & - & $4 / 0 / 0$ & 1 \\
\hline \hline
\end{tabular}

\section{Appendix B: Round-Robin Small-Size League}

This appendix includes all the results from the preliminary games in the smallsize league. The tables show the numbers of goals, the number of wins, losses, and draws $(\mathrm{W} / \mathrm{L} / \mathrm{D})$, and the rank of each team within each group. A win was counted against a team that forfeited a game.

\section{Group A}

- A1: AllBotz

- A2: Big Red

- A3: RobotIS

- A4: 5dpo

\begin{tabular}{||c||c|c|c|c|c|c||}
\hline \hline & A1 & A2 & A3 & A4 & W/L/D & Rank \\
\hline \hline A1 & - & $0-33$ & $0-35$ & $3-1$ & $1 / 2 / 0$ & 3 \\
\hline A2 & $33-0$ & - & $2-1$ & $8-0$ & $3 / 0 / 0$ & 1 \\
\hline A3 & $35-0$ & $1-2$ & - & $4-0$ & $2 / 1 / 0$ & 2 \\
\hline A4 & $1-3$ & $0-8$ & $0-4$ & - & $0 / 3 / 0$ & 4 \\
\hline \hline
\end{tabular}

\section{Group B}

- B1: Rogi2

- B2: CMUnited-99

- B3: Linked99

- B4: VUB (forfeited the games)

- B5: TPOTS

\begin{tabular}{||c||c|c|c|c|c|c||}
\hline \hline & B1 & B2 & B3 & B5 & W/L/D & Rank \\
\hline \hline B1 & - & $2-12$ & $5-0$ & $6-4$ & $3 / 1 / 0$ & 2 \\
\hline B2 & $12-2$ & - & $10-0$ & $4-0$ & $4 / 0 / 0$ & 1 \\
\hline B3 & $0-5$ & $0-10$ & - & $0-9$ & $1 / 3 / 0$ & 4 \\
\hline B5 & $4-6$ & $0-4$ & $9-0$ & - & $2 / 2 / 0$ & 3 \\
\hline \hline
\end{tabular}




\section{Group C}

- C1: Dynamo (forfeited the games after 4 goals suffered)

- C2: RoboRoos-99

- C3: SingPoly

- C4: Crimson

- C5: Owaribitos

\begin{tabular}{||c||c|c|c|c|c|c|c||}
\hline \hline & C1 & C2 & C3 & C4 & C5 & W/L/D & Rank \\
\hline \hline C1 & - & $0-4$ & $0-4$ & $0-4$ & $0-4$ & $0 / 4 / 0$ & 5 \\
\hline C2 & $4-0$ & - & $17-1$ & $10-0$ & $29-0$ & $4 / 0 / 0$ & 1 \\
\hline C3 & $4-0$ & $1-17$ & - & $5-2$ & $10-1$ & $3 / 1 / 0$ & 2 \\
\hline C4 & $4-0$ & $0-10$ & $2-5$ & - & $8-3$ & $2 / 2 / 0$ & 3 \\
\hline C5 & $4-0$ & $0-29$ & $1-10$ & $3-8$ & - & $1 / 3 / 0$ & 4 \\
\hline \hline
\end{tabular}

\section{Group D}

- D1: J-Star-99

- D2: FU-Fighters

- D3: LuckyStar

- D4: Microb3 (forfeited the games after 4 goals suffered)

\begin{tabular}{||c||c|c|c|c|c|c||}
\hline \hline & D1 & D2 & D3 & D4 & W/L/D & Rank \\
\hline \hline D1 & - & $1-10$ & $0-27$ & $4-0$ & $1 / 2 / 0$ & 3 \\
\hline D2 & $10-1$ & - & $2-1$ & $4-0$ & $3 / 0 / 0$ & 1 \\
\hline D3 & $27-0$ & $1-2$ & - & $4-0$ & $2 / 1 / 0$ & 2 \\
\hline D4 & $0-4$ & $0-4$ & $0-4$ & - & $0 / 3 / 0$ & 4 \\
\hline \hline
\end{tabular}

\section{Appendix C: Round-Robin Middle-Size League}

This appendix includes all the results from the preliminary games in the middlesize league. The tables show the numbers of goals, the number of wins, losses, and draws $(\mathrm{W} / \mathrm{L} / \mathrm{D})$, and the rank of each team within each group.

\section{Group A}

- A1: CS Freiburg

- A2: ISocRob

- A3: Raiders

- A4: GMD Robots

- A5: KIRC

- A6: Wisely

\begin{tabular}{||c||c|c|c|c|c|c|c|c||}
\hline \hline & A1 & A2 & A3 & A4 & A5 & A6 & W/L/D & Rank \\
\hline \hline A1 & - & $5-0$ & $6-0$ & $6-0$ & $6-0$ & $4-0$ & $5 / 0 / 0$ & 1 \\
\hline A2 & $0-5$ & - & $2-1$ & $1-0$ & $1-2$ & $1-1$ & $2 / 2 / 1$ & 3 \\
\hline A3 & $0-6$ & $1-2$ & - & $1-0$ & $0-1$ & $0-3$ & $1 / 4 / 0$ & 5 \\
\hline A4 & $0-6$ & $0-1$ & $0-1$ & - & $5-0$ & $0-4$ & $1 / 4 / 0$ & 5 \\
\hline A5 & $0-6$ & $2-1$ & $1-0$ & $0-5$ & - & $1-1$ & $2 / 2 / 1$ & 3 \\
\hline A6 & $0-4$ & $1-1$ & $3-0$ & $4-0$ & $1-1$ & - & $2 / 1 / 2$ & 2 \\
\hline \hline
\end{tabular}




\section{Group B}

- B1: Attempto!

- B2: NAIST

- B3: The Ulm Sparrows

- B4: RealMagiCol

- B5: 5dpo-2000

- B6: Matto

- B7: Alpha++

\begin{tabular}{||c||c|c|c|c|c|c|c|c|c||}
\hline \hline & B1 & B2 & B3 & B4 & B5 & B6 & B7 & W/L/D & Rank \\
\hline \hline B1 & - & $0-1$ & $0-0$ & $2-0$ & $2-0$ & $3-1$ & $0-1$ & $3 / 2 / 1$ & 4 \\
\hline B2 & $1-0$ & - & $0-3$ & $2-0$ & $3-0$ & $1-0$ & $0-5$ & $4 / 2 / 0$ & 3 \\
\hline B3 & $0-0$ & $3-0$ & - & $2-0$ & $3-0$ & $2-1$ & $0-1$ & $4 / 1 / 1$ & 2 \\
\hline B4 & $0-2$ & $0-2$ & $0-2$ & - & $0-2$ & $0-2$ & $0-2$ & $0 / 6 / 0$ & 7 \\
\hline B5 & $0-2$ & $0-3$ & $0-3$ & $2-0$ & - & $0-1$ & $0-2$ & $1 / 5 / 0$ & 6 \\
\hline B6 & $1-3$ & $0-1$ & $1-2$ & $2-0$ & $1-0$ & - & $0-5$ & $2 / 4 / 0$ & 5 \\
\hline B7 & $1-0$ & $5-0$ & $1-0$ & $2-0$ & $2-0$ & $5-0$ & - & $6 / 0 / 0$ & 1 \\
\hline \hline
\end{tabular}

\section{Group C}

- C1: Trackies-99

- C2: Agilo

- C3: ART-99

- C4: USC Dream Team-99

- C5: Patriarcas

- C6: Sharif CE

- C7: CoPS Stuttgart

\begin{tabular}{||c||c|c|c|c|c|c|c|c|c||}
\hline \hline & C1 & C2 & C3 & C4 & C5 & C6 & C7 & W/L/D & Rank \\
\hline \hline C1 & - & $1-0$ & $1-2$ & $2-1$ & $2-0$ & $1-2$ & $1-3$ & $2 / 4 / 0$ & 5 \\
\hline C2 & $0-1$ & - & $0-0$ & $1-0$ & $3-0$ & $2-1$ & $4-1$ & $4 / 1 / 1$ & 1 \\
\hline C3 & $2-1$ & $0-0$ & - & $1-0$ & $2-0$ & $1-0$ & $0-1$ & $4 / 1 / 1$ & 2 \\
\hline C4 & $1-2$ & $0-1$ & $0-1$ & - & $2-0$ & $0-10$ & $0-3$ & $1 / 5 / 0$ & 6 \\
\hline C5 & $0-2$ & $0-3$ & $0-2$ & $0-2$ & - & $0-4$ & $0-5$ & $0 / 6 / 0$ & 7 \\
\hline C6 & $2-1$ & $1-2$ & $0-1$ & $10-0$ & $4-0$ & - & $2-1$ & $4 / 2 / 0$ & 3 \\
\hline C7 & $3-1$ & $1-4$ & $1-0$ & $3-0$ & $5-0$ & $1-2$ & - & $4 / 2 / 0$ & 4 \\
\hline \hline
\end{tabular}

\section{Wild Card Match}

\begin{tabular}{||c||c|c|c|c|c||}
\hline \hline & ISocRob & NAIST & SharifCE & W/L/D & Rank \\
\hline \hline ISocRob & - & $1-2$ & $0-3$ & $0 / 2 / 0$ & 3 \\
\hline NAIST & $2-1$ & - & $0-1$ & $1 / 1 / 0$ & 2 \\
\hline SharifCE & $3-0$ & $1-0$ & - & $2 / 0 / 0$ & 1 \\
\hline \hline
\end{tabular}




\section{Appendix D: Round-Robin Sony Legged Robot League}

This appendix includes all the results from the preliminary games in the middlesize league. The tables show the numbers of goals, the number of wins, losses, and draws $(\mathrm{W} / \mathrm{L} / \mathrm{D})$, and the rank of each team within each group.

\section{Group A}

- A1: CM-Trio99 (Carnegie Mellon Univ., U.S.A.)

- A2: Humboldt Hereos (Humboldt Univ., Germany)

- A3: UNSW United (Univ. of New South Wales, Australia)

\begin{tabular}{||c||c|c|c|c|c||}
\hline \hline & A1 & A2 & A3 & W/L/D & Rank \\
\hline \hline A1 & - & $2-1$ & $3-0$ & $2 / 0 / 0$ & 1 \\
\hline A2 & $1-2$ & - & $1-2$ & $0 / 2 / 0$ & 3 \\
\hline A3 & $0-3$ & $2-1$ & - & $1 / 1 / 0$ & 2 \\
\hline \hline
\end{tabular}

\section{Group B}

- B1: BabyTigers-99 (Osaka Univ., Japan)

- B2: UPennalizers (Univ. of Pennsylvania, U.S.A.)

- B3: Team Sweden (Örebro Univ. and Stockholm Univ., Sweden)

\begin{tabular}{||c||c|c|c|c|c||}
\hline \hline & B1 & B2 & B3 & W/L/D & Rank \\
\hline \hline B1 & - & $2-0$ & $1-0$ & $2 / 0 / 0$ & 1 \\
\hline B2 & $0-2$ & - & $2-0$ & $1 / 1 / 0$ & 2 \\
\hline B3 & $0-1$ & $0-2$ & - & $0 / 2 / 0$ & 3 \\
\hline \hline
\end{tabular}

\section{Group C}

- C1: Les 3 Mousquetaires (LRP, France)

- C2: McGill RedDogs (McGill Univ., Canada)

- C3: Araibo (Univ. of Tokyo, Japan)

\begin{tabular}{||c||c|c|c|c|c||}
\hline \hline & C1 & C2 & C3 & W/L/D & Rank \\
\hline \hline C1 & - & $3-1$ & $2-0$ & $2 / 0 / 0$ & 1 \\
\hline C2 & $1-3$ & - & $0-1$ & $0 / 2 / 0$ & 3 \\
\hline C3 & $0-2$ & $1-0$ & - & $1 / 1 / 0$ & 2 \\
\hline \hline
\end{tabular}

\section{Appendix F: Finals}

This appendix includes the final results from the eliminatory games in the four leagues. The simulation league had a double-elimination tournament, while the other leagues had a single-elimination tournament. 


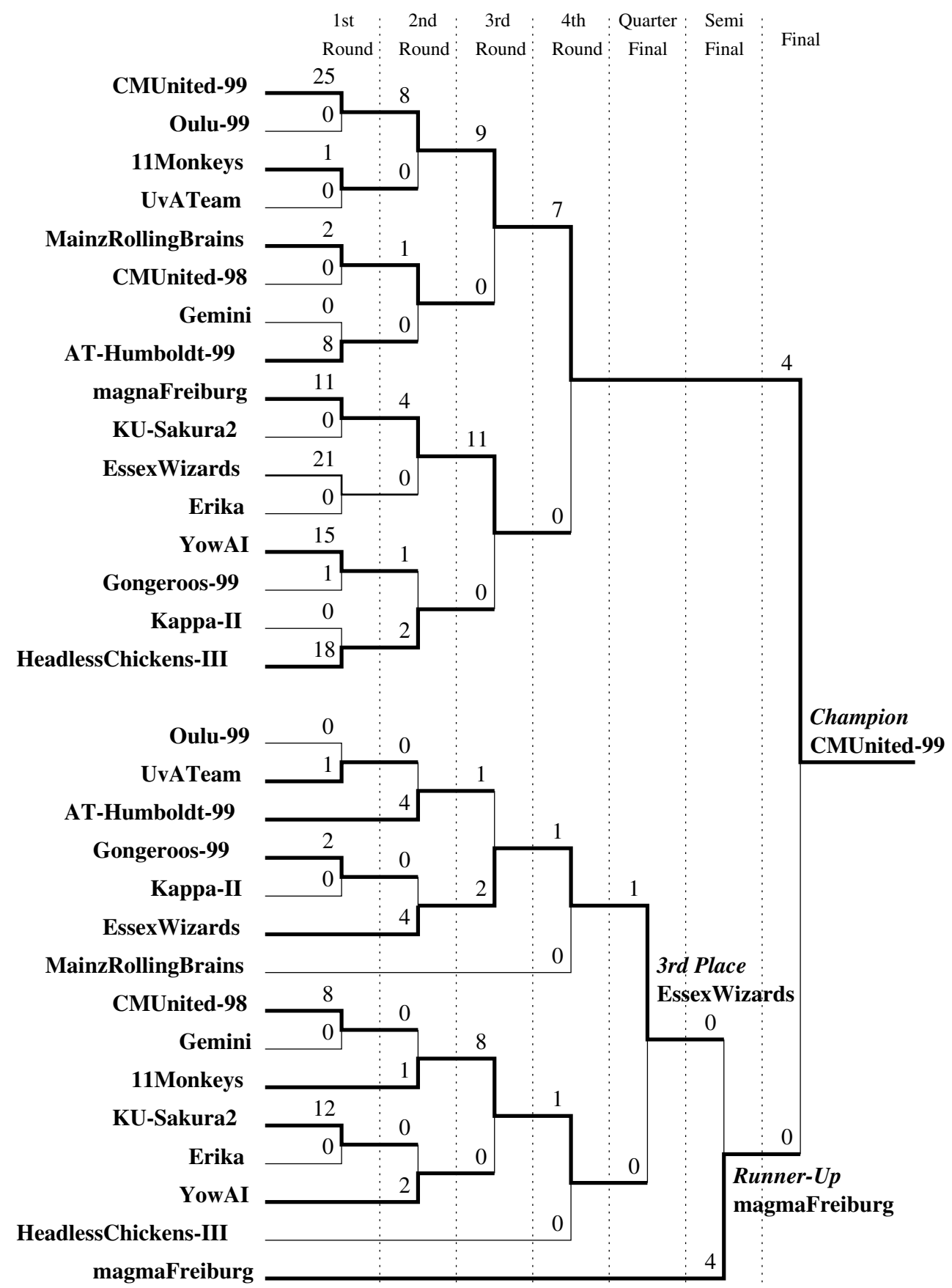

Fig. 7. Double-Elimination Tournament: Simulation League 


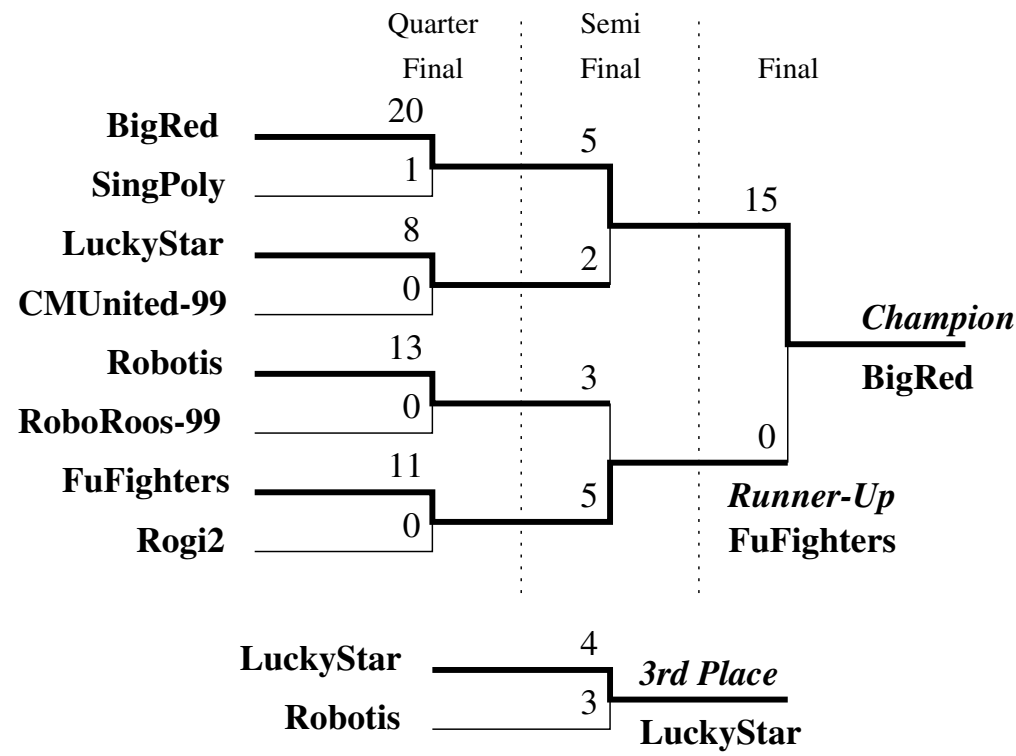

Fig. 8. Single-Elimination Tournament: F-180 Small-Size Robot League

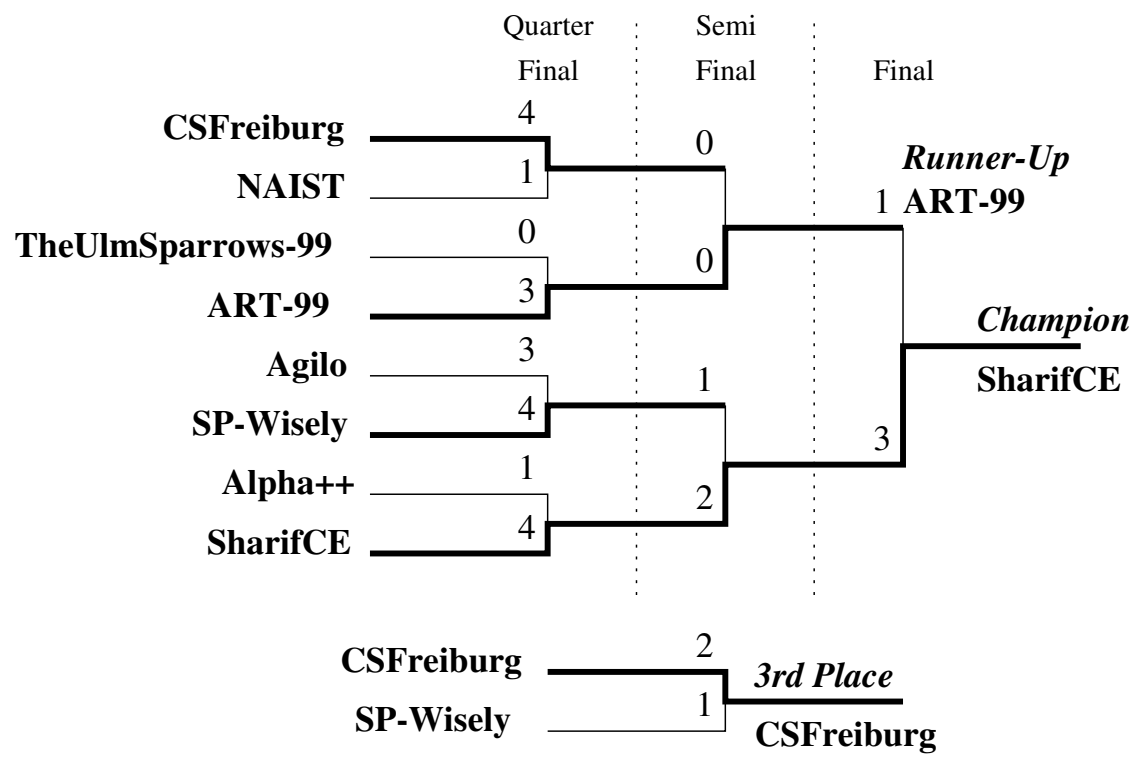

Fig. 9. Single-Elimination Tournament: F-2000 Middle-Size Robot League 

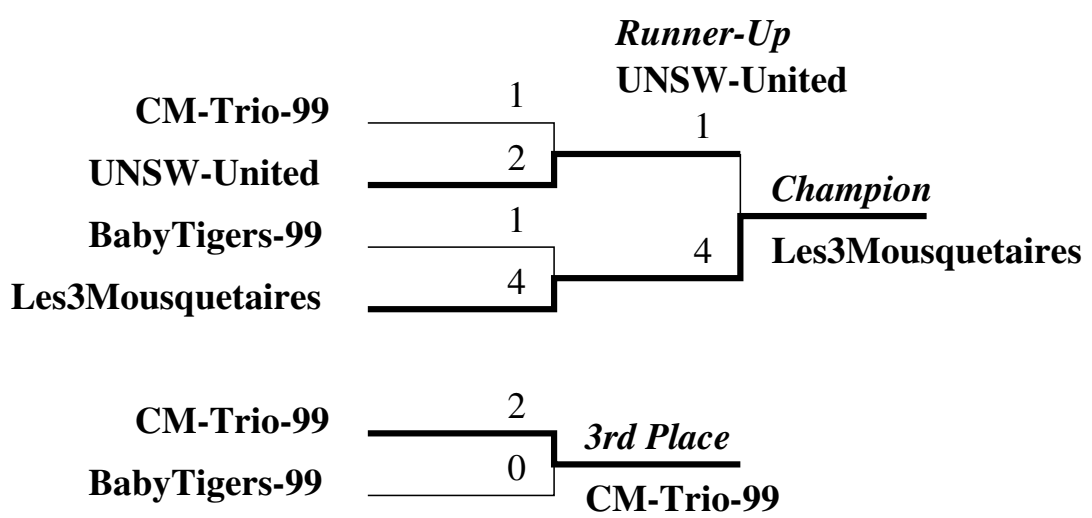

Fig. 10. Single-Elimination Tournament: Sony Legged Robot League

\section{References}

1. T. Andou. Refinement of soccer agents' positions using reinforcement learning. In Hiroaki Kitano, editor, RoboCup-97: Robot Soccer World Cup I, pages 373-388. Springer Verlag, Berlin, 1998.

2. E. Andre, G. Herzog, and T. Rist. Generating multimedia presentations for RoboCup soccer games. In Hiroaki Kitano, editor, RoboCup-97: Robot Soccer World Cup I, pages 200-215. Springer Verlag, Berlin, 1998.

3. M. Asada and H. Kitano, editors. RoboCup-98: Robot Soccer World Cup II. Springer, Lecture Notes in Artificial Intelligence, 1999.

4. K. Binsted. Character design for soccer commentary. In Minoru Asada and Hiroaki Kitano, editors, RoboCup-98: Robot Soccer World Cup II. Springer Verlag, Berlin, 1999 .

5. S. Coradeschi and J. Malec. How to make a challenging AI course enjoyable using the RoboCup soccer simulation system. In Minoru Asada and Hiroaki Kitano, editors, RoboCup-98: Robot Soccer World Cup II. Springer Verlag, Berlin, 1999.

6. H. Kitano, editor. RoboCup-97: Robot Soccer World Cup I. Springer, Lecture Notes in Artificial Intelligence, 1998.

7. H. Kitano, S. Suzuki, and J. Akita. RoboCup Jr.: RoboCup for Entertainment. In Proceedings of IEEE International Conference on Systems, Man, and Cybernetics 1999 (SMC-99), Tokyo, 1999.

8. H. Kitano, M. Tambe, P. Stone, M. Veloso, S. Coradeschi, E. Osawa, H. Matsubara, I. Noda, and M. Asada. The RoboCup synthetic agent challenge 97. In Proceedings of the Fifteenth International Joint Conference on Artificial Intelligence, 1997.

9. H. Lund and S. Pagliarini. RoboCup Jr. with Lego Mindstorms. In Proceedings of IEEE International Conference on Systems, Man, and Cybernetics 1999 (SMC-99), Tokyo, 1999.

10. H. Matsubara, I. Frank, K. Tanaka-Ishii, I. Noda, H. Nakashima, and K. Hasida. Automatic soccer commentary and RoboCup. In Minoru Asada and Hiroaki Kitano, editors, RoboCup-98: Robot Soccer World Cup II. Springer Verlag, Berlin, 1999.

11. I. Noda, H. Matsubara, K. Hiraki, and I. Frank. Soccer server: A tool for research on multiagent systems. Applied Artificial Intelligence, 12:233-250, 1998. 
12. A. Shinjoh. RoboCup-3D: The construction of intelligent navigation system. In Hiroaki Kitano, editor, RoboCup-97: Robot Soccer World Cup I, pages 188-199. Springer Verlag, Berlin, 1998.

13. P. Stone and M. Veloso. Task decomposition, dynamic role assignment, and lowbandwidth communication for real-time strategic teamwork. Artificial Intelligence, 110(2):241-273, June 1999.

14. I. Verner. RoboCup: A challenging environment for engineering education. In Minoru Asada and Hiroaki Kitano, editors, RoboCup-98: Robot Soccer World Cup II. Springer Verlag, Berlin, 1999. 